DOI: $10.22213 / 2658-3658-2017-37-42$

\title{
Photonic Applications and their Coexistence with Standard Data Transmissions
}

\author{
J. Radil, T. Horvath, P. Munster, O. Havlis, P. Skoda, V. Smotlacha, J. Vojtech \\ Optical Networks Department, CESNET z.s.p.o., Praha, Czech Republic \\ E-mail: jan.radil@cesnet.cz
}

Received: November 10, 2017

\begin{abstract}
Fibre networks are rather common today almost in many parts of the world and it looks that fibre as the transmission medium is here to stay for some time. Data transmissions in the backbone fibre networks are based on coherent detection principles and use multilevel phase modulations like quadrature phase shift keying used for $100 \mathrm{~Gb} / \mathrm{s}$ transmission speeds. But there are also new photonic applications with different requirements then raw speeds only. In order to guarantee seamless coexistence of standard and new photonic applications, we evaluate numerical simulations by using commercially available simulation tools. The simulations results show that such coexistence is possible when certain rules are followed. Simulation results are verified on real fibres to ensure reliable operation.
\end{abstract}

Keywords: fibre sensors, accurate time transfer, ultra stable frequency transfer, coherent transmission, high speed transmission.

\section{INTRODUCTION}

High speed data networks using standard single mode fibres (SSMF) are used to transfer huge amount of data between continents for good few years now. Coherent receiver principles and multilevel modulation formats can offer transmission speeds up to $400 \mathrm{~Gb} / \mathrm{s}$, with help of 64-level quadrature amplitude modulation (64QAM), in one dense wavelength division multiplexing (DWDM) channel.

But there are also other applications utilizing optical fibre called Photonic applications or services. Examples of such applications are accurate time transfer, ultra stable frequency transfer or fibre sensing and transmission speeds are not important anymore but rather minimal and constant delay or jitter and no vibrations.

National research and educational network (NREN) are pioneers of new developments in rather unexplored areas of research networking and special requirements for new applications were collected and described for example in [1] and [2].

\section{PHOTONIC (NON-DATA) APPLICATIONS}

New applications like accurate time transfer and ultra-stable frequency are not as exotic as they were just few years ago and their potential for sensing, metrology, navigation, seis-

(C) J. Radil, T. Horvath, P. Munster, O. Havlis, P. Skoda, V. Smotlacha, J. Vojtech, 2017 
mology or fundamental physics is accepted these days. Another fresh area of new applications is using of optical fibre for sensing.

Such non-data signals are usually amplitude modulated (or even not modulated at all which is the case for ultra-stable frequency transfer where the transmitted information is the frequency of photons) with rather slow speeds and new challenges certainly will arise when such non-data and high speed data signals are transmitted together in one fibre. And one fibre (or fibre infrastructure to be precise) must be shared by all such signals because it is not possible to deploy one fibre infrastructure for data, one for sensing applications and one for time and/or frequency transfer. The reasons are economical because no operator can afford to build and pay completely independent long haul fibre networks for every photonic application. This situation may be different in certain networking environments, for example Data Centre Interconnects (DCI) where optical cables with up to few thousands individual fibres may be installed and used independently.

Sensing (using fibre as a sensor) applications, for example phase sensitive optical time domain reflectometry (phi-ODTR) do use high optical powers which may influence phase modulated high speed data signals, especially via nonlinear mechanism of cross phase modulation (CPM). Phase sensitive OTDR is de facto slow amplitude modulated signal in its simplest form, known as on-off keying modulation (OOK). This is the reason that simultaneous transmission of such sensing signals is an important research area because coherent transmission systems can compensate for phenomenons like chromatic dispersion (CD) or even stochastic polarization mode dispersion (PMD) but today cannot cope with nonlinear effects like $\mathrm{CPM}$ or four wave mixing (FWM), described in detail in [3]. The reason that nonlinear effects cannot be compensated easily is complex mathematics of course.

It is clear that most demanding of photonic applications is sensing - because of relatively high optical powers launched into the fibre. Accurate time signals are also amplitude modulated but optical powers are similar to powers used for coherent data signals. And ultra stable frequency transfer is not modulated and also not as powerful as sensing signals, therefore possible mutual interference with data signals is relatively modest, however it is important to include them in practical verification because of optical amplifiers conditions like tampering with gain and output powers. Accurate time transfer results can be found in [4] and [5]. Ultrastable frequency transfer is described in [6] and [7]. Fibre sensing review can be found in [8] and some latest results in [9].

\section{PRACTICAL RESULTS}

For reasons mentioned in the previous section, simultaneous transmission of high speed coherent signals and high-power phase sensitive reflectometry signals, together with accurate time and ultra stable frequency, was performed in lab environment on fibre spools. We started experiments with shorter distances below $10 \mathrm{~km}$ of fibre and measurements were performed both with G.652 and G.655 fibres because both types of fibres are used in the CESNET network. Multiplexing and demultiplexing (or filtering) was realized on the standard $100 \mathrm{GHz}$ ITU grid. Some previous results can be found in [10] and [11].

Detailed measurement scheme showing both data and non-data applications is depicted in Figure 1. As can be seen, each service uses the wavelength according to the ITU DWDM $100 \mathrm{GHz}$ grid. The goal was to prove simultaneous transmissions in one fibre and with the standard channel spacing $100 \mathrm{GHz}$ (according to ITU). Requirement on the standard channel spacing is because in some cases is not possible to use whole spectrum but only dedicated 
DWDM channel for multiple services. Accurate time transfer $(1550.12 \mathrm{~nm})$, frequency transfer $(1552.52 \mathrm{~nm})$, and $100 \mathrm{G}$ data $(1551.72 \mathrm{~nm})$ were multiplexed together in a standard telecommunication multiplexer.

All multiplexed signals are sent to the phi-OTDR, then the sensing signal is mixed and all signals are launched into a fibre spool. This configuration allows loss minimization of the sensing signal.

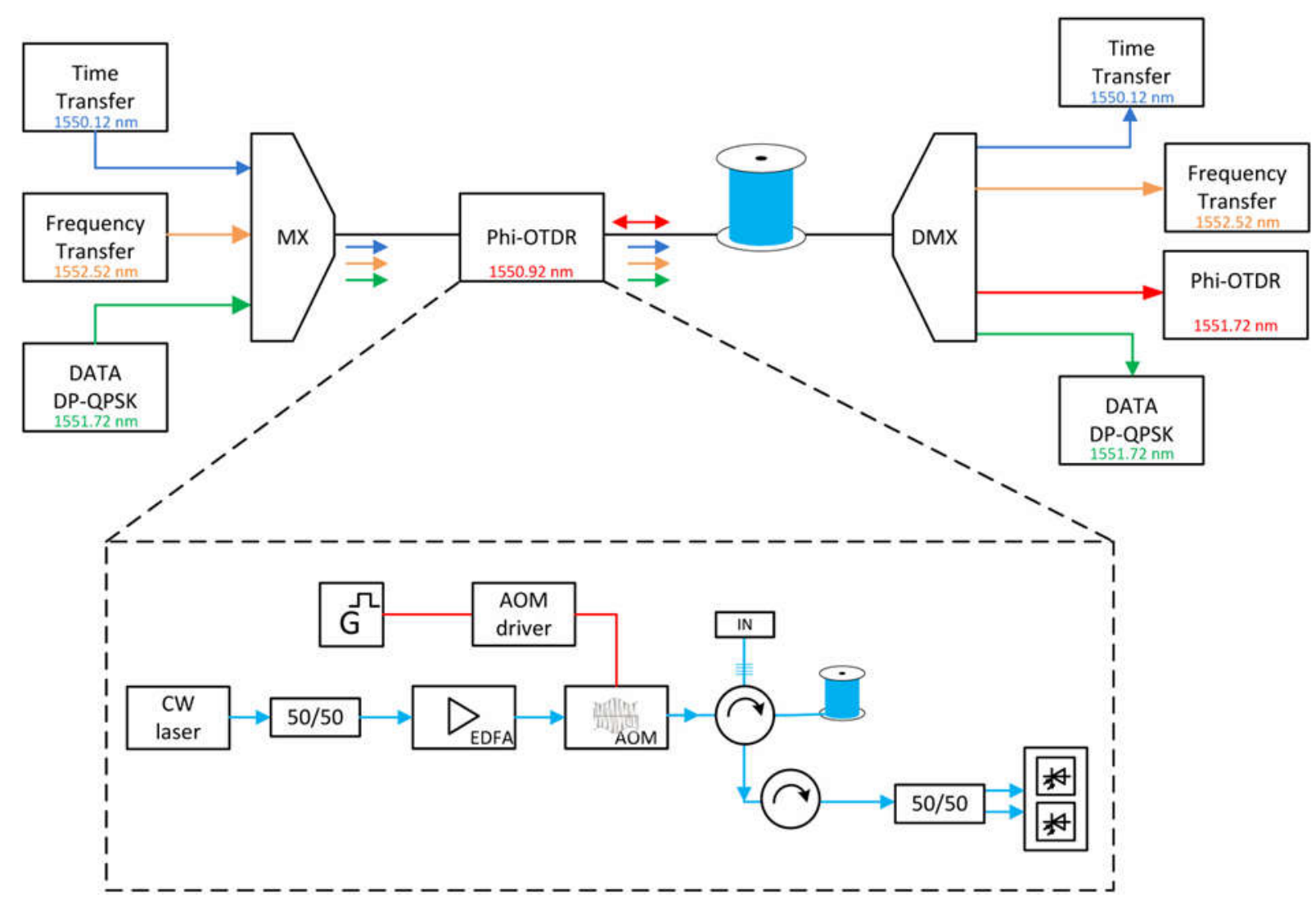

Figure 1. Set-up for laboratory measurements for accurate time, stable frequency, phi-OTDR and coherent signals

The experiments were performed for G.652 and G.655 fibres because such fibres are usually deployed in fibre optic networks - this is true for the CESNET backbone optical network. Some experiments were performed with G.653 fibre which may be considered as rather rare but we believe it is still useful for real comparison of three different fibres.

Important aspect was different duration of phi-OTDR pulses - $20 \mathrm{~ns}, 200 \mathrm{~ns}$ and $2000 \mathrm{~ns}$, with the same and constant repetition rate $5 \mathrm{kHz}$. As expected, G.652 fibre is more resistant to higher launched optical powers due to bigger effective core area.

Figure 2 and Figure 3 show results for three different types of fibres and dependency of bit error rate (BER) of $100 \mathrm{~Gb} / \mathrm{s}$ coherent data signal on pulsed sensing signals with different optical output powers launched into the fibre.

We can see that $200 \mathrm{~ns}$ and $2000 \mathrm{~ns}$ curves crossing for higher powers - this can be caused by small time intervals for measurements and also sensing pulses are sometimes overlapping with data signals more (it is a random process). G.653 has even smaller effective area compared to G.655 so tolerance to optical powers is smaller as can be seen on Figure 3. 


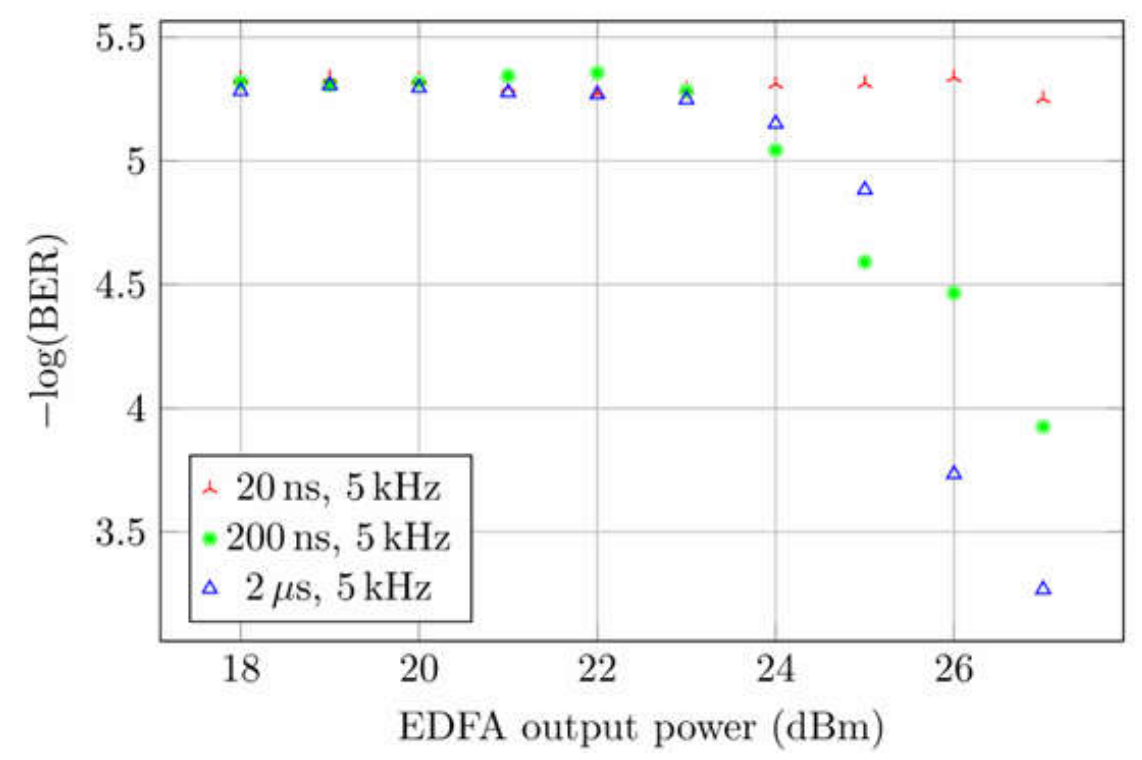

Figure 2. Dependence of $100 \mathrm{~Gb} / \mathrm{s}$ data signal on sensing signal for G.652+G.655 fibres

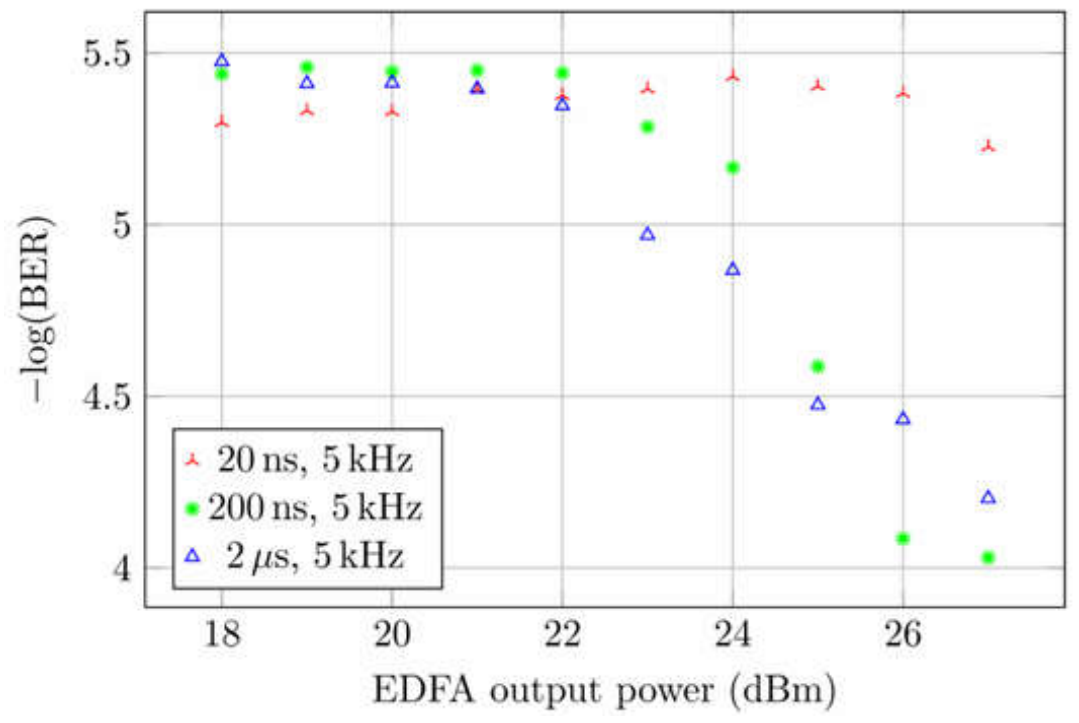

Figure 3. Dependence of $100 \mathrm{~Gb} / \mathrm{s}$ data signal on sensing signal for G.652+G.653 fibres

\section{NUMERICAL SiMULATIONS}

Numerical simulations are important part of this work. VPI photonics software was used to simulate optical data networks, sensing elements and transmission systems. Two different modulation formats (OOK, QPSK), three different transmission speeds $(1,10$ and $100 \mathrm{~Gb} / \mathrm{s}$ ) and various optical powers were used to verify nonlinear thresholds especially for CPM.

Different interferometric methods which are used for sensing applications were simulated. Three most important and also practically deployed methods use Mach-Zehnder, Michelson and Sagnac interferometres.

Two examples of simulations results are depicted in Figure 4 and Figure 5, showing the eye diagrams for $10 \mathrm{~Gb} / \mathrm{s}$ data signals and sensing pulse signals. As can be seen the difference between $50 \mathrm{GHz}$ and $150 \mathrm{GHz}$ adjacent-channel spacing is rather visible. 


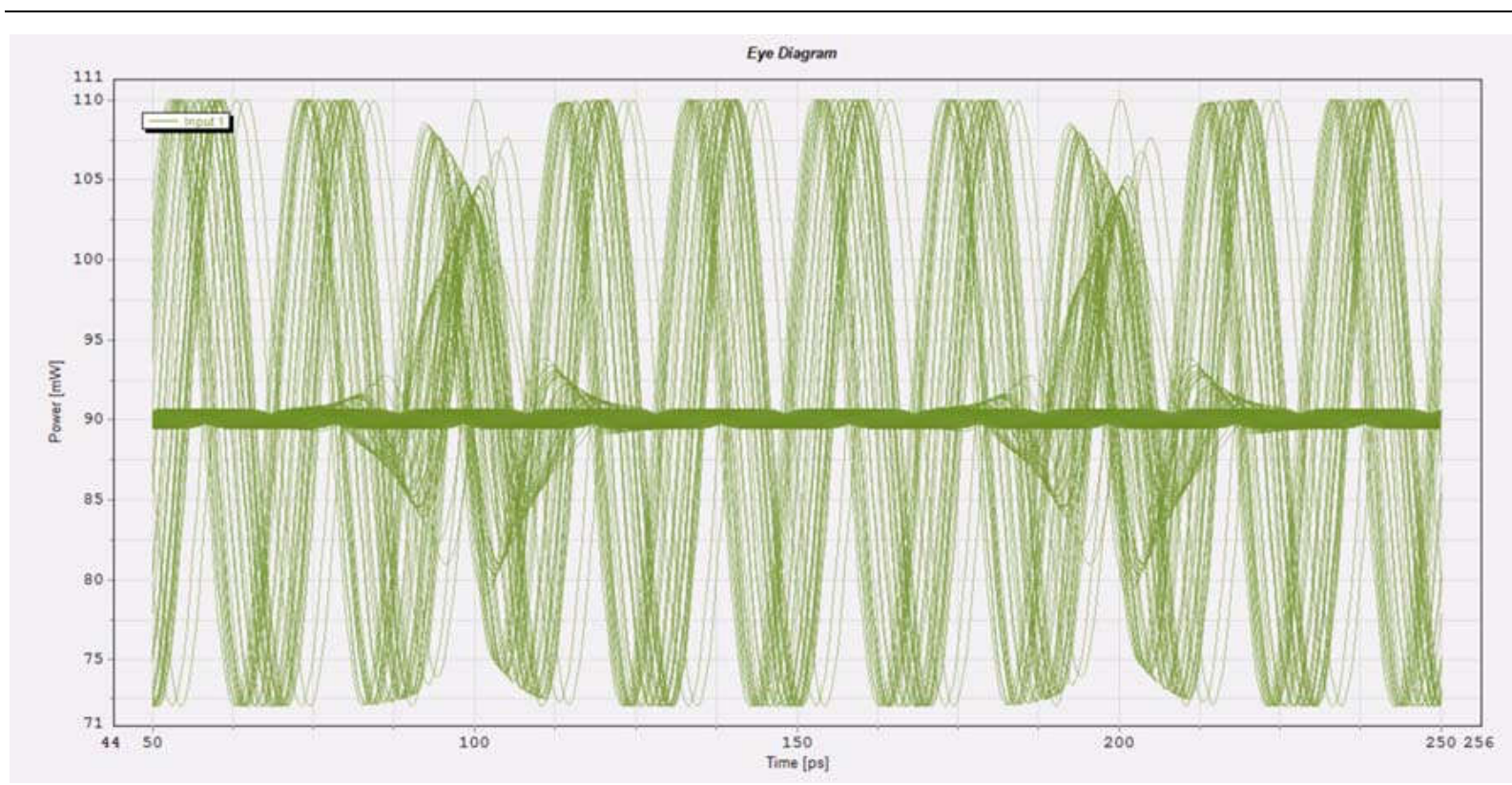

Figure 4. Eye diagrams for $10 \mathrm{~Gb} / \mathrm{s}$ and sensing signals for $50 \mathrm{GHz}$ channel spacing

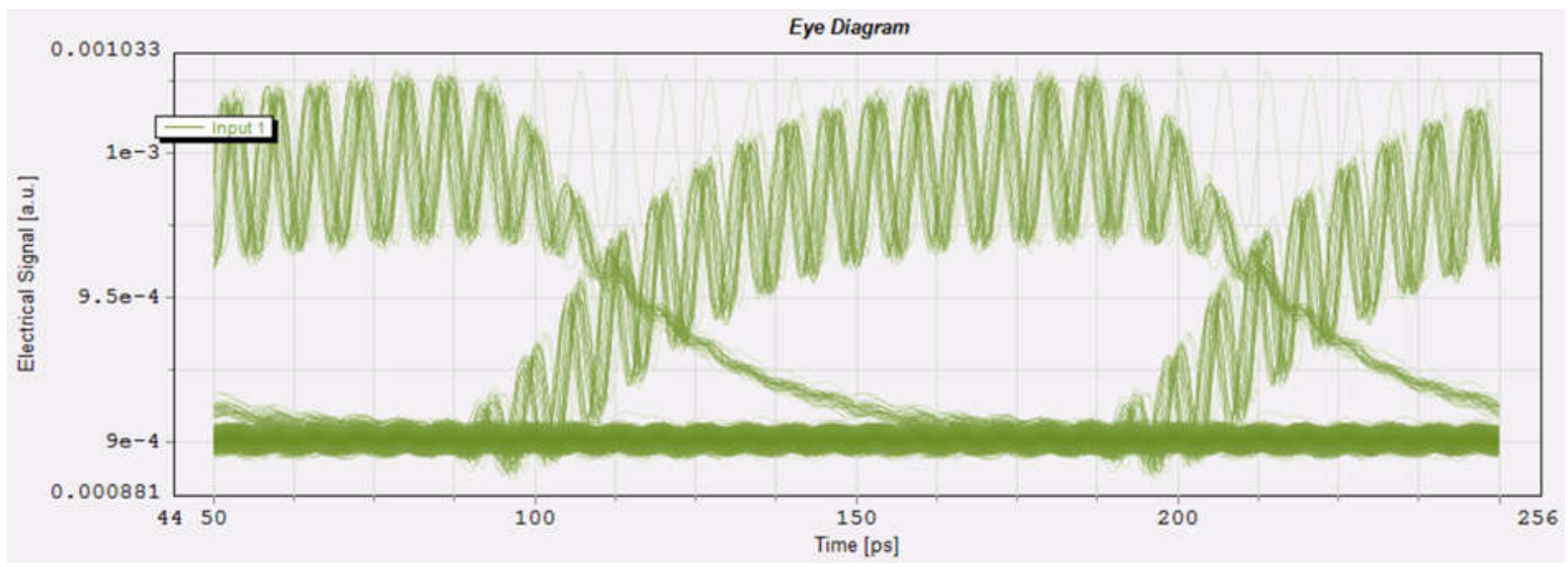

Figure 5. Eye diagrams for $10 \mathrm{~Gb} / \mathrm{s}$ and sensing signals for $150 \mathrm{GHz}$ channel spacing

\section{CONCLUSION}

We have verified the real coexistence of different applications in one fibre, based on previously performed numerical simulations. Verification was performed on two types of fibres deployed in the CESNET network - G.652 and G.655, with different pulse durations of phiOTDR sensing signals. Obviously that more powerful sensing pulses are, the worse degradation of data signals is observed.

We have found that real results measured on real optical systems are different than simulation results. One reason for this discrepancy can be that simulation software could not take into account all subtleties of all components used (e.g. phase difference for different fused fibre couplers). Also simulation software is designed mostly for data communications and not for special sensing applications.

But results indicate that simultaneous transmission of standard high speed coherent data signals and high-power sensing signals is possible without any serious consequences. This is very promising because such new sensing applications can be deployed not only in NRENs but also in commercial operational networks. 


\section{ACKNOWLEDGMENTS}

We would like to thank to colleagues from CESNET for useful discussions.

The work was supported by the project "E-infrastructure CESNET modernization", registration no. CZ.02.1.01/0.0/0.0/16_013/0001797, the National Sustainability Program under grant no. LO1401, and by the Ministry of Interior under grant no. VI20152020045.

\section{REFERENCES}

1. Altmanova, L. et al. (2013). Photonic services: challenge for users and for networkers. The GN3 project report.

2. Vojtech, J., Smotlacha, V., Skoda, P., Kuna, A., Hula, M., Sima, S. (2012). Photonic services, their enablers and applications. In Proceedings SPIE Volume 8516, Remote Sensing System Engineering IV, 85160H. doi:10.1117/12.929908.

3. Agrawal, G. P. (2010). Fiber-optic communication systems (4th ed.). New York, USA: Wiley.

4. Emardson, R., Hedekvist, P. O., Nilsson, M., Ebenhag, S.-C., Jaldehag, K., Jarlemark, P., Rieck, C., Johansson, J., Pendrill, L. R., Lothberg, P., \& Nilsson, H. (2008). Time transfer by passive listening over a 10-Gb/s optical fiber. IEEE Transactions on Instrumentation and Measurement, 57(11), 2495-2501. doi:10.1109/TIM.2008.925023.

5. Zhang, H., Wu, G., Hu, L., Li, X., \& Chen, J. (2015). High-precision time transfer over 2000-km fiber link. IEEE Photonics Journal, 7(6), 1-9, doi:10.1109/JPHOT.2015.2499541.

6. Predehl, K., Grosche, G., Raupach, S. M. F., Droste, S., Terra, O., Alnis, J., Legero, T., Hänsch, T. W., Udem, T., Holzwarth, R., \& Schnatz, H. (2012). A 920-kilometer optical fiber link for frequency metrology at the 19th decimal place. Science, 336(6080), 441-444. doi:10.1126/science.1218442.

7. Lopez, O., Haboucha, A., Chanteau, B., Chardonnet, C., Amy-Klein, A., \& Santarelli, G. (2012). Ultra-stable long distance optical frequency distribution using the Internet fiber network. Optics Express, 20(21), 2351823526. doi:10.1364/OE.20.023518.

8. Thévenaz, L. (2006). Review and progress on distributed fibre sensing. In Optical Fiber Sensors 2006, OSA Technical Digest (CD), paper ThC1. Cancun, Mexico: Optical Society of America. doi:10.1364/OFS.2006.ThC1.

9. Schenato, L., Palmieri, L., Camporese, M., Bersan, S., Cola, S., Pasuto, A., Galtarossa, A., Salandin, P. \& Simonini, P. (2017). Distributed optical fibre sensing for early detection of shallow landslides triggering. Scientific Reports, 7(article number: 14686), 1-7. doi:10.1038/s41598-017-12610-1.

10. Munster, P., Horvath, T., Havlis, O., Vojtech, J., Radil, J., Velc, R., \& Skaljo, E. (2017). Simultaneous transmission of standard data, precise time, stable frequency and sensing signals and their possible interaction. In Proceedings of SPIE, 10231, Optical Sensors 2017, 102312A. doi:10.1117/12.2266240.

11. Vojtech, J., Slapak, M., Skoda, P., Radil, J., Havlis, O., Altmann, M., Munster, P, Velc, R., Kundrat, J., Altmannova, L., Vohnout, R., Horvath, T., Hula, M., Smotlacha, V., Cizek, M., Pravdova, L., Rerucha, S., Hrabina, J., \& Cip, O. (2017). Joint accurate time and stable frequency distribution infrastructure sharing fiber footprint with research network. Optical Engineering, 56(2), 027101. doi:10.1117/1.OE.56.2.027101. 